\title{
SIMULATION OF T-JOINTS BETWEEN RHS STEEL MEMBERS WITH OFFSET IN ABAQUS CAE
}

\author{
SVITLANA KALMYKOVA
}

\begin{abstract}
Czech Technical University in Prague, Faculty of Civil Engineering, Department of Steel and Timber Structures, Czech Technical University in Prague, Thákurova 7, 16629 Prague 6, Czech Republic

correspondence: svitlana.kalmykova@fsv.cvut.cz
\end{abstract}

\begin{abstract}
The current paper focuses on numerical simulation peculiarities of offset welded rectangular hollow section joints. Understanding the modelling techniques can result in easier and faster and above all correct outcomes from FEA for future use. The steel joints under discussion are composed from cold-formed regular rectangular hollow sections where RHS brace members are laterally shifted from chord axis. Joints work under monotonically increasing compression load applied to a brace top. Numerical models were developed in FE programme Abaqus. FE-models is composed of C3D8R 8-noded solid linear brick elements with an emphasis on mesh size effect and modelling of a weld seam. FE advanced model were compiled considering both material and geometric nonlinearities. For validation purposes, the full-scale laboratory tests were conducted. Proposed FE models reliably predict the structural behaviour of welded offset T-joints thanks to good agreement achieved on deformation limit $3 \% b_{0}$ with the maximum deviation $10.3 \%$.
\end{abstract}

KEYWORDS: FE programme Abaqus, Hollow structural sections (HSS), numerical modelling, offset T-joints, rectangular hollow sections (RHS), truss connections.

\section{INTRODUCTION}

Steel structural hollow sections, including rectangular hollow sections (RHS), are nowadays one of the most efficient load-bearing elements of the lattice structures. Despite its wide-spread application, there is still lack of design techniques for joints produced with offsets.

Since the mid-1960s, vast study on HSS connections structural behaviour has been undertaken. The results were implemented in current regulations and standards such as EN 1993-1-8 (2005), ANSI/AISC 360-05 (2005), CSA-S16-09 (2009), ISO 14346:2013 or a design guide CIDECT DG3. It is essential to note that existing methods of HSS connections design are for coaxial ones only, i.e. centreline of a branch member should have an intersection with centreline of a chord. The term "Standard" is used for geometry of aforesaid connections. At the same time, the question concerning mechanical resistance of joints under discussion is practically uncovered by design recommendations and guidelines.

As of today, there are few studies published on the topic, namely, the earliest experimental research of laterally offset T-and N-joints done by Dawe et al. [1], Kalmykova and Wald [2, 3] including laboratory and numerical research, and Wei and Packer [4] who performed a set of the offset SHS T- and X- joints laboratory testing and proposed certain design approaches simultaneously with an analytical equation to predict resistance of the offset joints.

The strength, modes of failure and loaddisplacement curves can be gained from a proper numerical analysis. Considering costliness of physical laboratory experiments, it is of high impotence to find ways of effective numerical simulation of joints (see Figure 1) to trustworthy predict their structural behaviour.
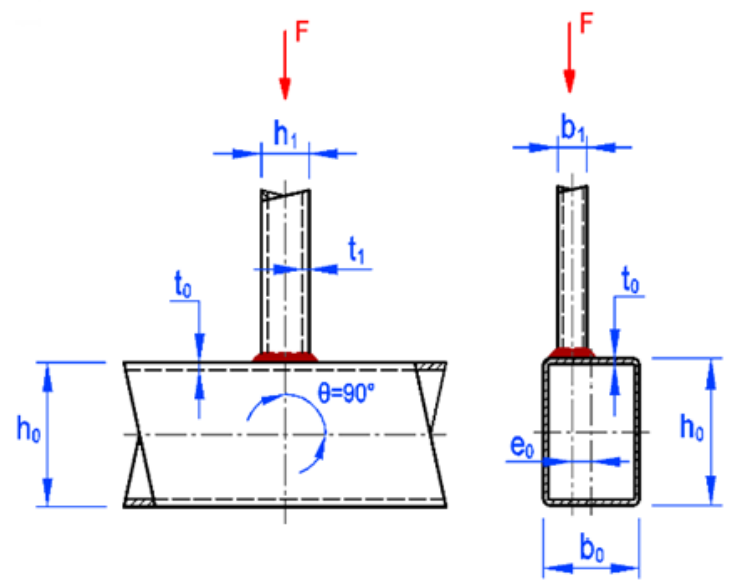

Figure 1. Welded offset T-joint geometry.

The aim of the current study is to present a practical numerical simulation guideline for offset joints and connections using Abaqus CAE.

\section{Finite ELEMENT MODELLingS}

\subsection{General}

Numerical modelling of the fully welded offset Tjoints was done in ABAQUS. It is general-purpose finite element programme, release 6.14. FE models of joints were composed in compliance with prEN 

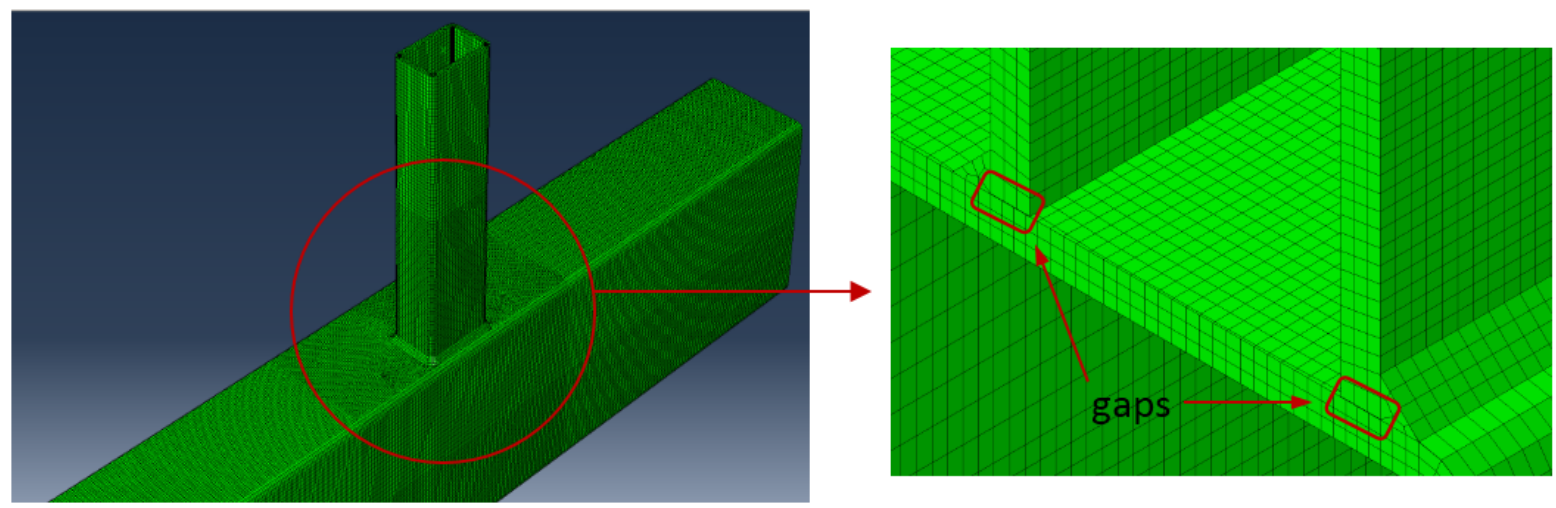

Figure 2. Finite element mesh of T-joint.

1993-1-14: 2020. STATIC, RIKS procedure available in the ABAQUS library was employed to run loaddisplacement nonlinear analysis. FE models of offset joints were built considering both geometric and material nonlinearities. Carefully performed sensitivity study allowed to define the certain element type, FE mesh size of finite elements as well as welding material property. It brings accurate results with adequate computational costs. Boundary conditions, loading, weld seam modelling and simulation of materials are fundamental inputs in performed FE analysis.

\subsection{GEOMETRY OF JOINTS}

Experimental programme includes two types of Tjoints employed for the validation procedure. The first type presents a regular coaxial geometry of $\mathrm{T}$ joint without any offset; the second one was produced with the brace lateral offset (see Figure 1). Both joints were loaded by the monotonically increased axial compression applied at the brace top.

Brace member is made of cold-formed rectangular hollow section RHS $50 \times 30 \times 4 \mathrm{~mm}$ EN10219. Coldformed RHS $150 \times 100 \times 4 \mathrm{~mm}$ EN10219 is used for chord member.

\subsection{Element TyPeS AND FE MESH SIZE IN A BRICK MODEL}

The element type used for joint modelling is 3D 8node solid element with reduces integration (C3D8R) from Abaqus library. It is fully isoperimetric element with the first order interpolation. Finite element mesh of a model is presented in Figure 2. It consists of two regions with two various FE element sizes. Smaller mesh region comprises FE bricks with the sizes up to $4 \times 4 \times 4 \mathrm{~mm}$ and is distributed along the direct chord axis on the distance of $240 \mathrm{~mm}$. The distance of smaller mesh region for the brace member along the direct brace axis equals $300 \mathrm{~mm}$. The rest of the FE mesh consists of $8 \times 8 \times 8 \mathrm{~mm}$ elements for both members of the joint. Additionally, at the corner areas, the finer 4-element mesh was employed because of great importance to transfer stresses from a flange to a web of a joint member. Isoparametric angles were monitored as there was no distortions in the FE bricks. Aspect ratio of the FE bricks were limited to be less than 3 .

The number of in-depth FE layers for chord and brace walls were monitored in order to analyse model sensitivity. The number of layers were varied from 1 to 6 . Based on the obtained data and subsequent analysis, 3-layer mesh was chosen for further simulations by virtue of ability to provide accurate results with reasonable computational costs.

Apart from C3D8R brick FE units, two more types of solid 3D elements were utilized. There are C3D8I and C3D20R. It is to check processing time and precision of obtained results for FE models made by it.

\subsection{LOADING AND WELD SIMULATION}

Joints work under monotonically increasing compression load applied at a brace top. Axial compression was imposed to the reference point on an analytical rigid plate (see Figure 3 ).

Weld seam, in general, has considerable impact to the mechanical resistance of T-joints. This is particularly the case for the fillet weld corner areas. Thus, weld was employed in FE models of offset joints. As for the brick model, weld seam is the only member that joining brace and chord. Thereto, a yawns or gaps were modelled between external face of the chord top flange and bottom section of the brace with the help of advanced simulation on initial geometry creation step. Specific procedure was applied to the meshing. As for the shell model, one should go to the section 2.7.

For simplicity, the material properties of the weld seam and steel members were assumed the same. It is due to complexity of weld material property investigation when an electrode's composition is mixed with the main material of a joint. FE solid elements types coinside with the chosen type for a model.

\subsection{BOUNDARY CONDITIONS}

As for boundary conditions of the joint, the chord member was stacked on the rigid base, i.e. it is fully restrained from rotation and displacement. Additionally, the displacement in the direction of the applied 


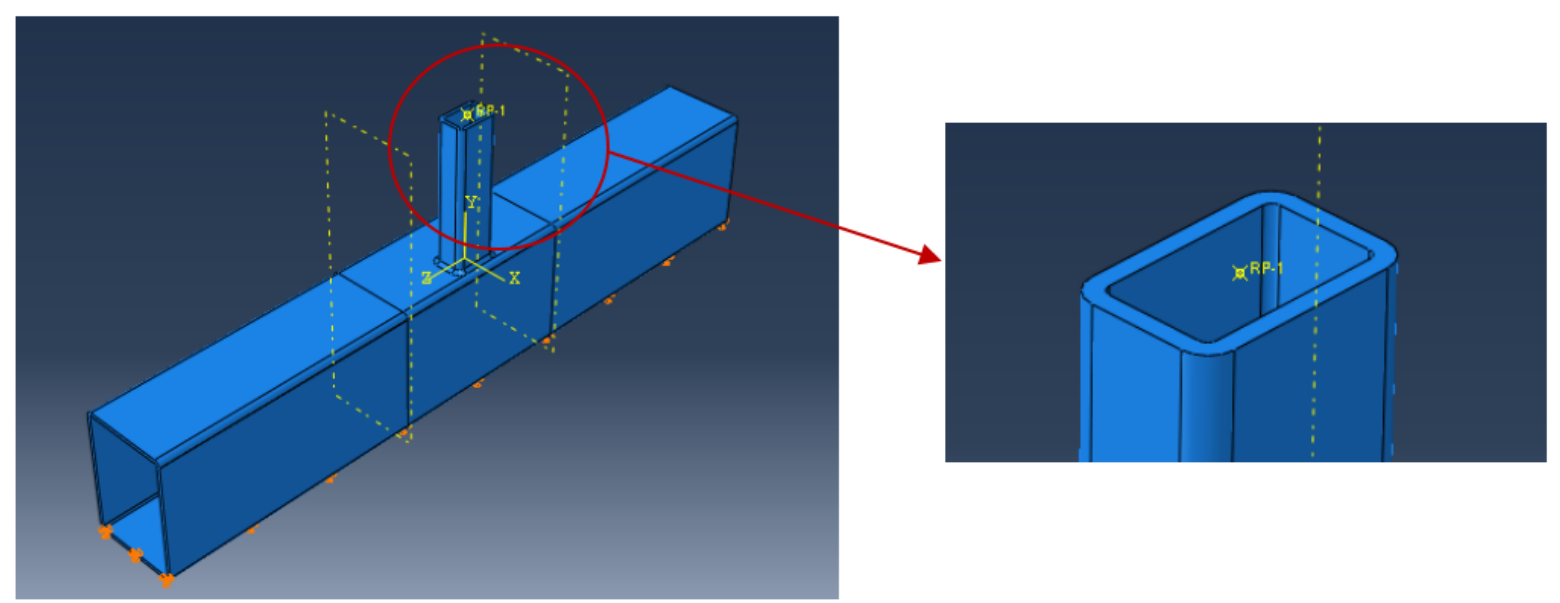

FiguRE 3. Displacement of the reference point and boundary conditions on the model.

load is allowed for the top brace edge, while other displacements and rotations are prohibited.

\subsection{MATERIAL}

Tensile coupon tests (see Figure 4) were performed in order to provide real material properties to an advanced FE model. The coupons were cut in the rolling direction from additional pieces of RHS made from the same material. A total of 5 tensile tests were conducted: 3 tests on S355 J2H material taken from a chord element section, and 2 tests on S355 J2H taken from a brace element profile. The middle part of a profile face was chosen for cutting coupons to avoid zones affected by hardening. Material properties including Young's modulus, the ultimate tensile stress and the corresponding strain were recorded.

More detailed information including measured data and true stress-strain curves is provided in Kalmykova and Wald [2].

In general, for elastic-plastic bodies analysed by Abaqus, material properties of a specimen are assigned in two dialog boxes, namely, mechanical elastic and plastic material behaviour dialog boxes.

The elastic response of steel is modelled by linear elasticity with Young modulus. Elastic properties are specified as isotropic (see Table 1). To model isotropic plasticity in ABAQUS, the Mises yield surface is used. The plasticity data are determined by true stress versus logarithmic plastic strain (see Table 2).

\begin{tabular}{ccc}
\hline Fracture Strain & Stress Triaxiality & Strain Rate \\
\hline 1 & 0.3 & 0 \\
0.3 & 1 & 0 \\
\hline
\end{tabular}

TABlE 1. Material behavior assigned in Abaqus (Elastic Isotropic).

For the specimen modelling in Abaqus, the real material model resulting from the coupon tensile tests was used. The initial part illustrates proportional

\begin{tabular}{cc}
\hline Yield Stress & Plastic Strain \\
\hline 320 & 0 \\
355.594 & $2 \mathrm{E}-005$ \\
365.568 & 0.01806 \\
420 & 0.04679 \\
459.563 & 0.07013 \\
492.25 & 0.09297 \\
517.5 & 0.11532 \\
537.625 & 0.1372 \\
567 & 0.17962 \\
587.5 & 0.22035 \\
\hline
\end{tabular}

TABle 2. Material behavior assigned in Abaqus (Plastic Isotropic).

dependency of stress and strain, an evidence of elastic property, up to the tensile yield strength $f_{y}$ with recorded Young's modulus $E$ and Poisson's ratio $\nu$. Beyond the elastic limit, ultimate tensile strength $f_{u}$ and elongation after fracture $\varepsilon_{f}$ measured on coupon tests create the post-yield response of the bilinear material model. Additionally, Von-Mises yield rule and kinematic hardening model were employed into the model.

Extensive plastic strain is involved into nonlinear FE analysis. For that reason, static stress-strain diagram measured on coupon tests was converted to true stress-logarithmic plastic strain diagram. ABAQUS user's manual contains recommendations based on which the true stress $\sigma_{\text {true }}$ and logarithmic plastic strain $\varepsilon_{p l}$ were computed.

During the simulation, an attempt to simulate the elimination from calculations of individual physically damaged finite elements was made since the strength criterion for such elements is no longer satisfied. Damaged cells were excluded from the calculation by introducing the ductile damage option (see Table 3). 

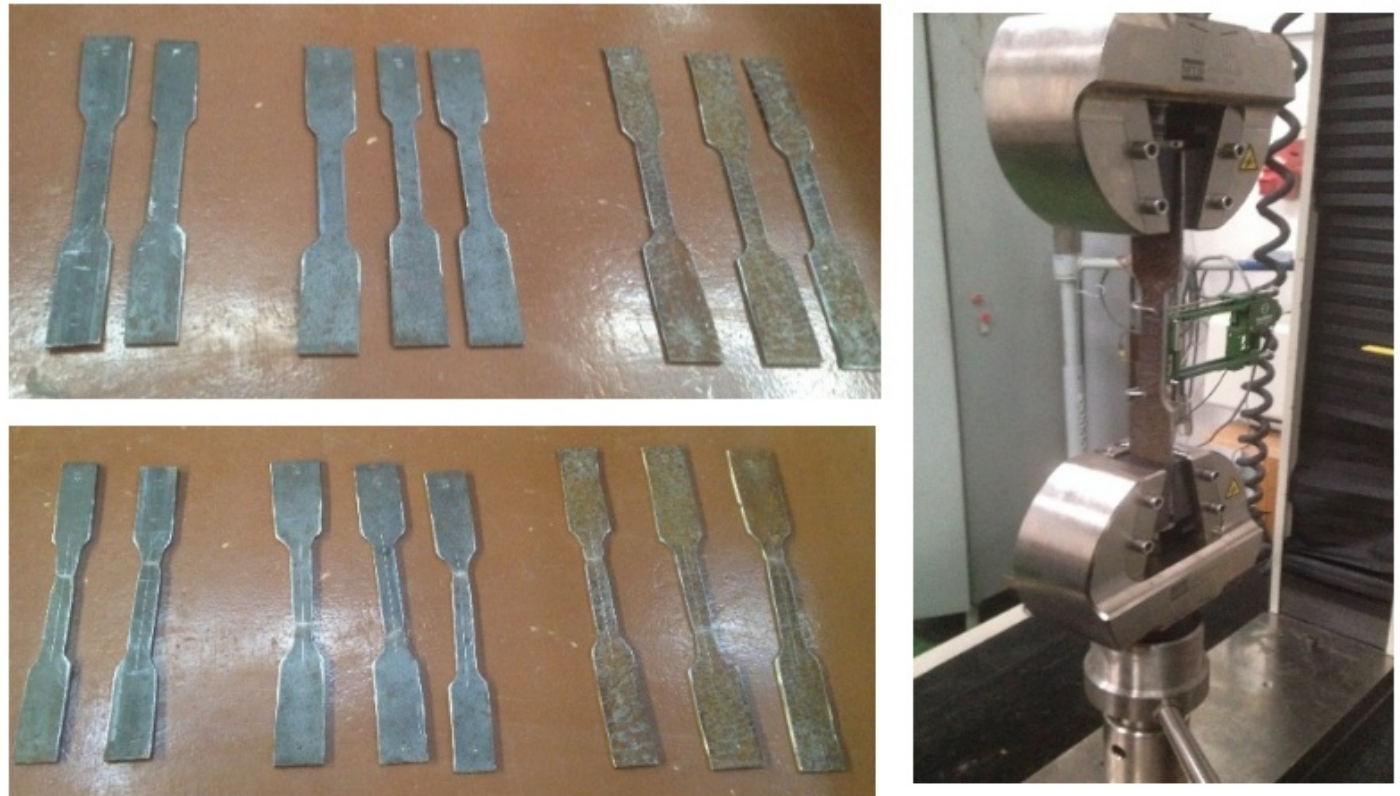

Figure 4. Coupon tests specimens and scheme of loading.

\begin{tabular}{cc}
\hline Young's Modulus & Poisson's Ratio \\
\hline 201000 & 0.3 \\
\hline
\end{tabular}

TABLE 3. Material behavior assigned in Abaqus (Ductile Damage).

\subsection{SHELl FE MODEL}

In addition to the main procedure of numerical model validation, it was decided to conduct comparative analysis of similar FE-models composed by solid elements (C3D8R) and their counterparts constructed by shell finite elements.

The element type used for joint modelling is a 4node doubly curved shell element with reduced integration (S4R) from Abaqus library. A 5-point integration was utilized by the thickness of the shell with all degrees of freedom per node. In modelling, sections of the joints' brace and chord were presented by their middle surfaces with assigned thicknesses which are correspondent to the real thicknesses of components. As it was stated in more earlier studies (Korol and Mirza [5], Matos et al. [6]), shell finite elements are able to assure precise computation to overwhelming majority of applications owing to transverse shear deformation presence that crucial for thick shell elements simulation (see Figure 5).

A four-node doubly curved shell element with reduced integration (S4R) has been used to model brace and chord members. A five-point integration was applied through the shell thickness with a full complement of six degrees of freedom per node. In modelling, sections of the joints' brace and chord were presented by their middle surfaces with thicknesses which are correspondent to the real thicknesses of components. As it was stated in more earlier studies
(Korol and Mirza [5], Matos et al. [6]), shell elements provide an accurate solution to most applications by allowing for transverse shear deformation, which is important for simulation of thick shell element Figure 5 .

As a simplification, some of researchers omit the weld seam in shell FE models of welded HSS joints. This usually creates undervaluing of the joint strength. Nevertheless, for the specimens under discussion, the weld seam was considered. Filled weld with throat thickness of $10 \mathrm{~mm}$ at the intersection of brace and chord was modelled according to van der Vegte et al. [7]. Inclined at an 45 angle circle of elements is displaced along the brace and chord intersection area.

Some of the researchers omit the welds for simplicity in the modelling of welded tubular joints using shell elements, which leads to underestimating the joint strength. However, for the specimens under discussion, the weld seam was considered. Filled weld with throat thickness of $10 \mathrm{~mm}$ at the intersection of brace and chord was modelled according to van der Vegte et al. [7] using a "ring" of shell element along the brace and chord intersection region.

\section{EXPERIMENTAL STUDY}

Full-scale laboratory experiments were carried out by Kalmykova and Wald [2] with the aim to study failure modes of the standard and offset T-joints specimens and to get data for the validation of FE models developed for numerical studies.

\section{VALidation}

For the purposes of this paper, results from numerical FE advanced model in Abaqus were calibrated against relevant full-scale experimental data for both 


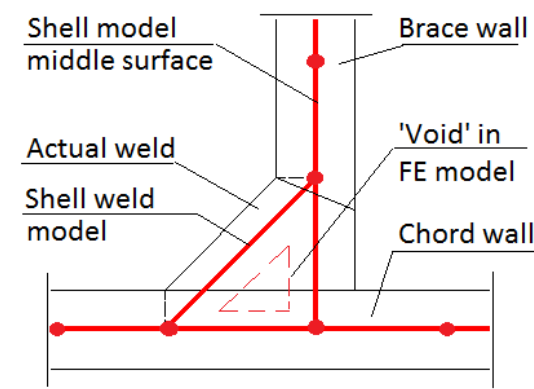

FiguRE 5. "Shell" FE models weld seam.

standard and laterally offset T-joints. Abaqus FE models were validated against experimental results conducted by the authors mentioned hereinabove. Validation procedure and its complete outcomes have been provided in detail in [3]. The comparison of the "load-displacement" diagrams coming from the fullscale experiments and FE analysis is shown on Figure 6 and Figure 7.

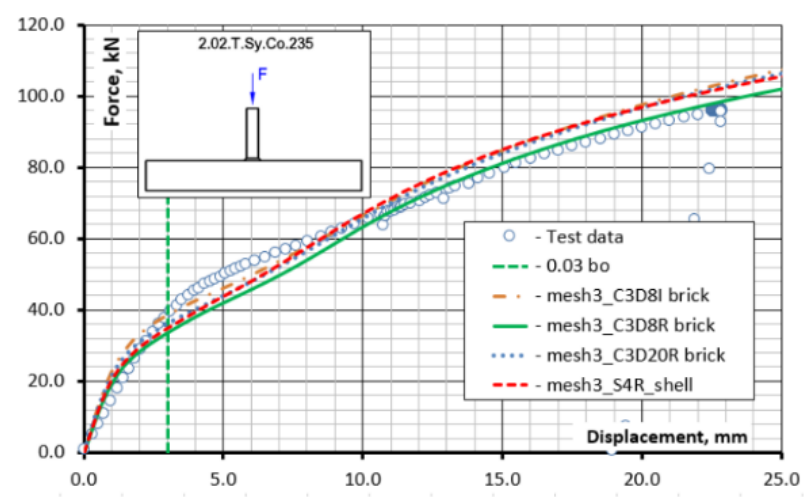

Figure 6. Validation of FE models against laboratory tests for a standard joint.

Test and finite element analysis results show good agreement achieved for both standard and offset models on allowed deformation level of $0.03 b_{0}$ with the maximum difference of $10.3 \%$ and the minimum difference of $2.6 \%$.

\section{Conclusion}

FE models are an essential tool for structural behaviour assessment of steel offset joints between HSS members. The structural response predicted by these numerical models adequately reflects the actual behaviour of the joints.

To guarantee it, the FE models were validated on the results of physical laboratory tests. The FE analyses results accurately aligned with the test outcomes. Hence, it could be said that the proposed FE models reliably predict the structural behaviour of welded offset T-joints with RHS members.

3D 8-node solid elements with reduces integration (C3D8R) is recommended for further simulation of offset T-joints between RHS members as the ones

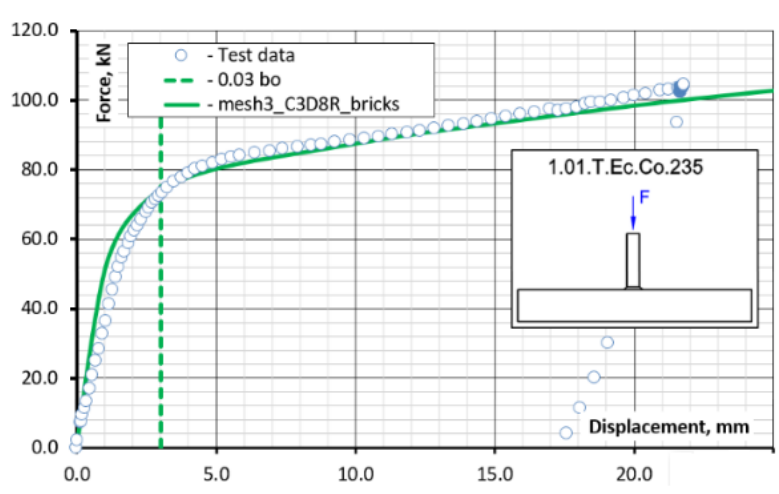

FiguRE 7. Validation of FE models against laboratory tests for an offset joint.

that provide accurate results in adequate computation time.

\section{List of Symbols}

$\begin{array}{ll}E & \text { Young's modulus } \\ \nu & \text { Poisson's ratio } \\ \sigma_{u} & \text { ultimate tensile stress } \\ \varepsilon_{u} & \text { ultimate strain } \\ f_{y} & \text { tensile yield stress } \\ f_{u} & \text { ultimate tensile strength } \\ \sigma_{t r u e} & \text { true stress } \\ \varepsilon_{p l} & \text { logarithmic plastic strain }\end{array}$

\section{ACKNOWLEDGEMENTS}

The research was carried out under the grant SGS19/150/OHK1/3T/11.

\section{REFERENCES}

[1] J. L. Dawe, Y. Liu, R. Zhang, A. Dukuze. Experimental study of offset hss connections. Journal of Structural Engineering, American Society of Civil Engineers 132(5):717-725, 2006.

[2] S. Kalmykova, F. Wald. Full-scale experimental study of welded rhs t-joints with offset and its main outcomes. Grant journal 8(1):76-81, 2019.

[3] S. Kalmykova, F. Wald. Welded t-joints between rectangular hollow section members with offset. In Proceedings of XII Conference on Steel and Composite Construction 1:1157-1167.

[4] F. Wei, J. A. Packer. Experimental study of laterally offset rhs x-connections.

[5] R. M. Korol, F. A. Mirza. Finite element analysis of rhs t-joints. Journal of the Structural Division 108(9):2081-2098, 1982.

[6] R. M. M. P. Matos, L. F. Costa-Neves, L. R. O. Lima, et al. Resistance and elastic stiffness of rhs " $t$ " joints: Part i - axial brace loading. Latin American Journal of Solids and Structures 12:2159-2179, 2015.

[7] G. J. Van der Vegte, C. H. M. de Koning, Puthli, et al. Numerical simulation of experiments on multiplanar tubular steel x-joints. International Journal of Offshore and Polar Engineering 1(3), 1991. 